\title{
The stability of drop shapes for translation at zero Reynolds number through a quiescent fluid
}

\author{
C. J. Koh and L. G. Leal \\ Chemical Engineering Department, California Institute of Technology, Pasadena, California 91125
}

\begin{abstract}
(Received 19 January 1989; accepted 21 April 1989)
Boundary-integral calculations are used to investigate the evolution of the shape of an initially nonspherical drop that translates at zero Reynolds through a quiescent, unbounded fluid. For finite capillary numbers, it is shown that the drop reverts to a sphere, provided the initial deformation is not too large. However, drops that are initially deformed to a greater extent are shown to deform continuously, forming an elongated shape with a tail when initially prolate, and a flattened shape with a cavity at the rear when initially oblate. The critical degree of deformation decreases as the capillary number increases and appears to be consistent with the results of Kojima et al. [Phys. Fluids 27, 19 (1984) ], who showed that the spherical drop is unstable to infinitesimal disturbances in the limit $\mathrm{Ca}=\infty$.
\end{abstract}

\section{INTRODUCTION}

The motion of a viscous drop translating under the action of buoyancy through an unbounded, quiescent fluid is a classical problem with a very long history in fluid mechanics. A solution in the creeping flow limit was obtained many years ago, attributed to Hadamard-Rybzynski, and based upon the assumption that the shape is spherical. Later, Taylor and Acrivos ${ }^{1}$ considered small inertia effects and showed that the drop deforms slightly to an oblate ellipsoid shape for small Reynolds number, but that the sphere is an exact steady solution for arbitrary capillary number in the limit $R e \equiv 0$. The stability of the steady, spherical shape to infinitesimal disturbances of shape was studied more recently by Kojima et $a l^{2}$ These authors showed that the sphere was a stable solution for arbitrarily small disturbances of shape for all finite capillary numbers, but was unstable in the limit $\mathrm{Ca}=\infty$. The instability was predicted to appear at the rear of the drop as a growing tail for an initially prolate shape, and as a growing indentation or cavity for an initially oblate shape, with a characteristic growth rate that scales as $(1+\lambda)^{-1}$ with changes in the viscosity ratio $\lambda$ (i.e., $\left.\lambda \equiv \mu_{\text {drop }} / \mu_{\text {suspending fluid }}\right)$. In contrast, the magnitude of the initial disturbance at the front of the drop was predicted by the linear theory to decay rapidly to zero, so that the front of the drop returns rapidly to a hemispherical shape. The obvious question that we pursue here is whether the stability of the spherical shape for nonzero capillary numbers carries over to finite initial deformations.

This investigation is based upon numerical solutions of the free boundary, creeping-flow problem of an initially deformed drop rising through a quiescent fluid, using the wellknown boundary-integral method. The particular implementation of this technique is very similar to that described in recent publications from our group, cf. Stone and Leal. ${ }^{3}$ Since the method is well known and the details have already been described elsewhere, we simply pose the problem and describe the results in the present paper, with only a few remarks to establish those details of the numerics that would be necessary to reproduce the results that are reported here (e.g., the number of surface elements). A preliminary de- scription of the work described here was contained in the conference proceedings of a recent meeting on bubble and drop dynamics. ${ }^{4}$

\section{PROBLEM FORMULATION}

We consider the translational motion of a viscous drop through an unbounded fluid in the creeping flow approximation. The two fluids are Newtonian, immiscible, and the interface is characterized by a finite and constant interfacial tension. Thus the governing equations and boundary conditions are

$$
\begin{aligned}
& -\nabla p_{1}+\nabla^{2} \mathbf{u}_{1}=0 \\
& -\nabla p_{2}+\nabla^{2} \mathbf{u}_{2}=0 \\
& \mathbf{u}_{2} \rightarrow 0, \quad \text { as }\|\mathbf{x}\| \rightarrow \infty \\
& \mathbf{u}_{1}=\mathbf{u}_{2}, \quad \text { at drop interface, } \\
& \lambda \mathbf{n} \cdot \mathbf{T}_{1}-\mathbf{n} \cdot \mathbf{T}_{2} \\
& \quad=-(\mathbf{n} / \mathrm{Ca}) \boldsymbol{\nabla} \cdot \mathbf{n}-\mathbf{n} z\left[3\left(1+\frac{3}{2} \lambda\right) /(1+\lambda)\right],
\end{aligned}
$$$$
\text { at drop interface }
$$

(subscript 1 denotes the drop phase and 2 denotes the suspending phase), where we have nondimensionalized the velocity with respect to the translational velocity of a spherical drop of the same volume, i.e.,

$$
u_{c}=\frac{1}{3} \frac{a^{2} g}{v_{2}}\left|\frac{\rho_{1}}{\rho_{2}}-1\right|\left(\frac{1+\lambda}{1+\frac{3}{2} \lambda}\right) .
$$

The stresses are nondimensionalized with respect to $\mu_{1} u_{c} / a$ in fluid 1 and $\mu_{2} u_{c} / a$ in fluid 2 .

In the present case, the shape of the drop is specified initially as nonspherical and we are interested in calculating the evolution of drop shapes as a function of time after the initial moment. The drop is assumed to translate under the action of a constant body force. Hence, as the shape of the drop changes, the translational velocity changes, in accord with the change in hydrodynamic mobility. Our primary interest is to determine whether the shape of an initially nonspherical drop returns to a sphere or deforms further as a 
function of time. In the latter case, we say that the initial drop shape is unstable.

The problem formulated above is solved by the boundary-integral method. As stated earlier, the implementation of this method for our current work is essentially identical to the description contained in the earlier publication of Stone and Leal. ${ }^{3}$ In the interest of conserving space, we thus refer to this earlier work for a description of the numerical details. In this present work, we typically use 50 ring-shaped elements on the surface of the drop that is axisymmetric in shape, but this number is varied, depending upon the evolution of shapes and the need for adequate resolution in regions of large curvature.

\section{RESULTS}

First of all, in order to check the numerical code, we compute the velocity of a rising spherical drop and compare the results with the Hadamard-Rybzynski solution. Typically, the computed velocity on each of the surface elements is within at least $0.05 \%$ of the exact solution when the surface of the drop is divided into 50 elements. Specifically, this accuracy is attained for the cases of $\lambda=0.1,1.0$, and 5.0, and is improved when using more surface elements.

In the present study, our primary objective is an investigation of the evolution of drop shapes as a function of the degree of initial deformation for a number of fixed, nonzero values of the capillary number $\mathrm{Ca}$. A set of typical results is shown in Fig. 1 for a fixed initial shape, $\lambda=0.5$, and various $\mathrm{Ca}$. Here, the initial shape is characterized by the parameter $\Delta=\frac{1}{3}$, where $\Delta \equiv(L-B) /(L+B)$, and $L$ corresponds to the length along the axis of rotation of the ellipsoid, as shown in Fig. 2. Using this definition, a prolate ellipsoid has a positive $\Delta$ value while an oblate ellipsoid has a negative $\Delta$ value. The drop shape in Fig. 1 is shown at several specific points in time (nondimensionalized by $a / u_{c}$ ). The position of the drop is incremented in the figure by the exact amount of its

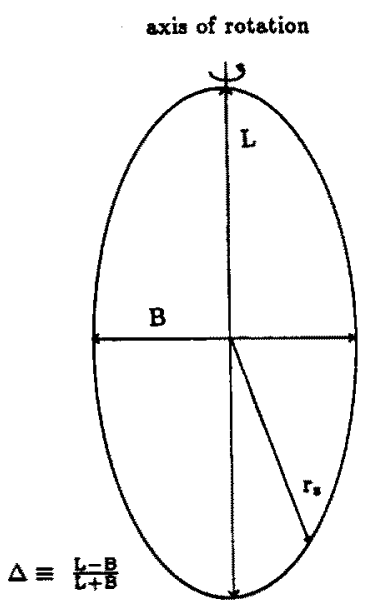

FIG. 2. A sketch showing the definition of $\Delta$.

numerically calculated displacement. What we can see, qualitatively, from Fig. 1, is that the drop returns to a steady spherical shape for $\mathrm{Ca} \leqslant 1.25$; but for this initial shape, the deformation increases continuously in time for $\mathrm{Ca} \geqslant 1.5$, with the front of the drop returning to a hemispherical shape, while the rear of the drop develops a tail that increases in length as a function of time. Eventually, in the cases shown in Fig. 1, the tail begins to pinch and it appears that the drop will break as the tail separates from the main parent drop (though, of course, the boundary-integral method can only be pushed to a very thin neck, but cannot be carried to the point of actual pinch-off). Thus, for $\lambda=0.5$ and $\Delta=\frac{1}{3}$, $\mathrm{Ca}_{\text {crit }}$ lies between 1.25 and 1.5.

If we consider other values of $\Delta$, similar results are obtained. In particular, solutions for initially prolate shapes are shown in Fig. 3 for $\Delta=\frac{1}{21}$ and $\lambda=0.5$, and in Fig. 4 for $\Delta=0.6$ and $\lambda=0.5$. Again, we see that for small enough $\mathrm{Ca}$, the drop returns to a steady spherical shape, but above
$\mathrm{Ca}=1.25$

$\mathrm{Ca}=1.5$

$\mathrm{Cs}=\mathbf{2 . 0}$
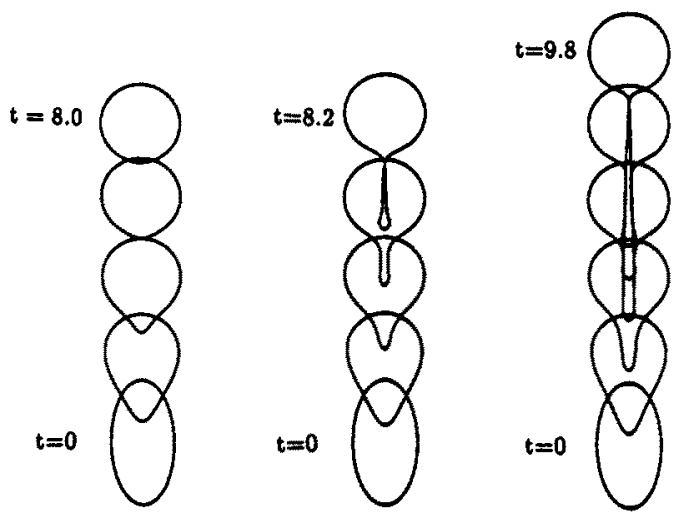

$\lambda=0.5$

$$
\Delta \equiv \frac{t-8}{t+8}=\frac{1}{3}
$$

FIG. 1. Evolution of prolate drops with same initial shapes but different $\mathrm{Ca}$. The time between intermediate shapes $=2.0$.
$\mathrm{Ca}=4.5$

$\mathrm{Cs}=\mathbf{5 . 0}$

$\mathrm{Cs}=8.0$
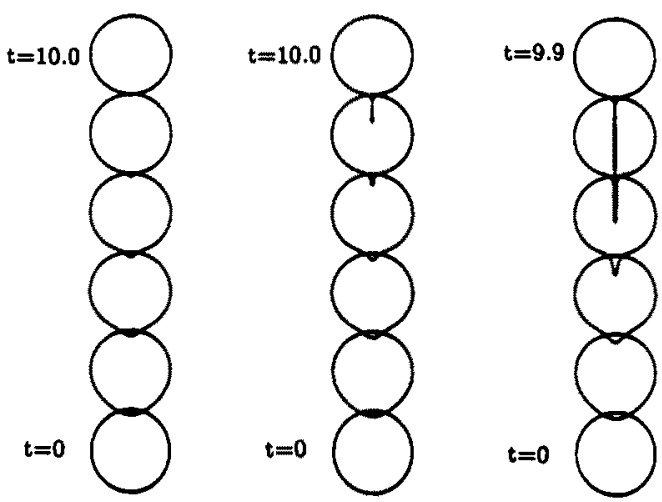

$\lambda=0.5$

$\Delta \equiv t+\frac{1}{t+1}$

FIG. 3. Evolution of prolate drops with same initial shapes but different Ca. The time between intermediate shapes $=2.0$. 
$\mathrm{t}=6.0$

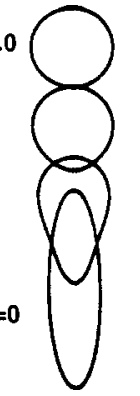

$\lambda=0.5$

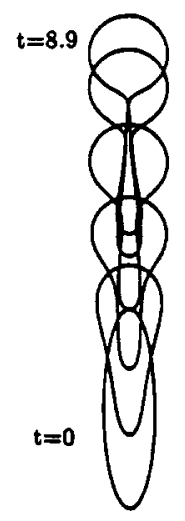

$\Delta \equiv \frac{\operatorname{ton}}{t+8}=0.6$
FIG. 4. Evolution of prolate drops with same initial shapes but different $\mathrm{Ca}$. The time between intermediate shapes $=2.0$.

some critical value of $\mathrm{Ca}$ the drop develops an increasingly elongated tail with an increase of time. Comparison of Figs. 1,3 , and 4 shows, however, that the critical Ca for instability increases as $\Delta$ is decreased. This would seem qualitatively consistent with the prediction from the linear stability theory that the drop is unstable to infinitesimal disturbances for $\mathrm{Ca}=\infty$, but is stable to such disturbances for finite values of Ca. It may also be noted that the present predictions for finite $\mathrm{Ca}$ are qualitatively similar to the linear stability theory for $\mathrm{Ca}=\infty$, in the sense that the initially prolate shape is predicted, for $\mathrm{Ca}>\mathrm{Ca}_{\text {crit }}$, to develop a tail at the rear and a hemispherical shape at the front.

We have also carried out a similar investigation of the magnitude of initial deformation for a series of oblate initial shapes for several fixed values of the capillary number. Typical numerical results for this series of numerical experiments are represented in Fig. 5. Qualitatively, the stability behavior is similar to that demonstrated above for initially prolate shapes. Specifically, for each nonzero capillary number, there is a critical initial deformation beyond which the drop shape is unstable, in the sense that the drop does not return to a spherical steady state, but continues to deform with an increase in time. As in the case of prolate initial shapes, we have found that as the degree of initial deformation increases, $\mathbf{C a}_{\text {crit }}$ decreases. However, instead of a tail, the initially oblate shape develops a cavity at the rear. As before, the front of the drop rapidly reverts to a hemispherical shape for all of the initial conditions that we have considered.

It may be noted that the formation of a cavity from an initially oblate shape is again qualitatively consistent with the predictions of the linear stability theory. In fact, a comparison of the solution given by Kojima et al. ${ }^{2}(\lambda=5$, $\mathrm{Ca}=\infty)$ and our numerical solution $(\lambda=5, \mathrm{Ca}=100)$ is shown in Fig. 6. We can see that the evolution of the drop shape is very similar. The difference is attributed to the difference in $\mathrm{Ca}$ and the assumption of Kojima et al. of small deviation from sphericity.

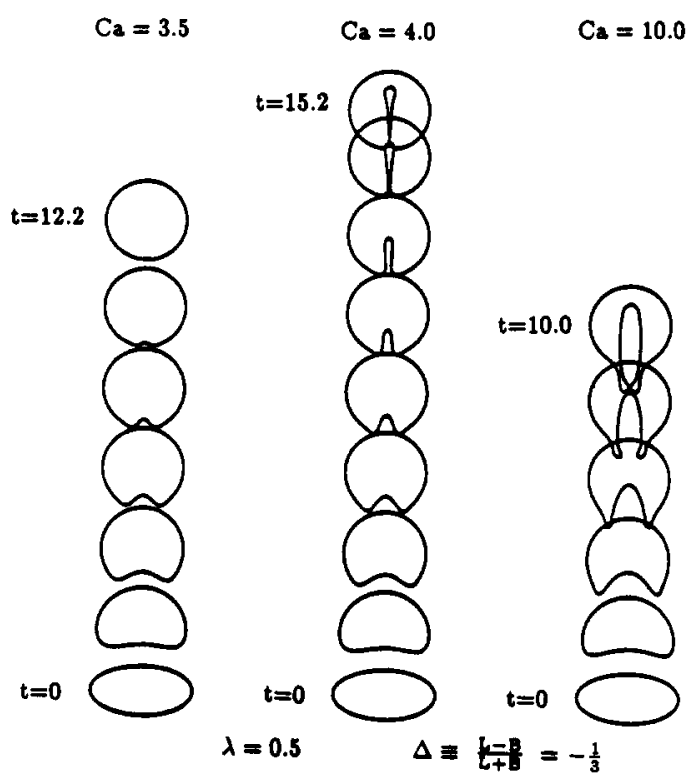

FIG. 5. Evolution of oblate drops with same initial shapes but different $\mathrm{Ca}$. The time between intermediate shapes $=2.0$.

Several other details of the problem described above can be examined easily via the boundary-integral technique. One factor that we have considered is the role of the viscosity ratio $\lambda$. We illustrate one representative case in Fig. 7, where we show results for a fixed value of $\Delta=\frac{1}{3}$ and a fixed $\mathrm{Ca}=2.0$, but two values of $\lambda=0.5$ and 5.0, respectively. The results shown in Fig. 7 are not plotted at equal values of time $t$, as in the previous figures, but at equal values of $\bar{t} \equiv t /$ $(1+\lambda)$, which is the scaling with $\lambda$ that is suggested by the linear stability analysis. We see that the results are initially almost identical, thus corroborating the prediction from the linear theory that the viscosity ratio enters primarily via the time scale of deformation in the unstable case. Not surpris-

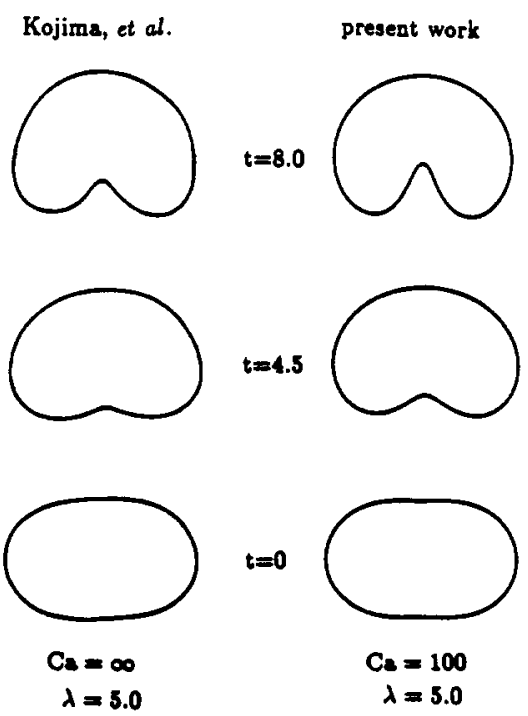

FIG. 6. Comparison of the present work with the results of Kojima et al. ${ }^{2}$ 


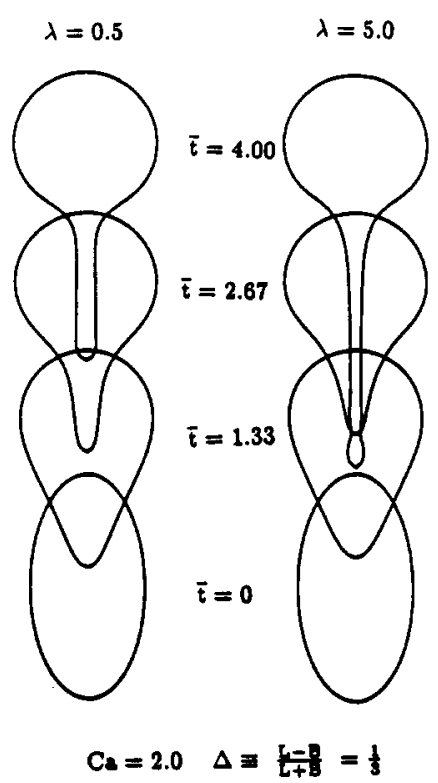

FIG. 7. Using the scaling $\bar{t} \equiv t /(1+\lambda)$, as suggested by the linear stability analysis, the drop shapes are almost identical, especially during the initial stage of the deformation.

ingly, however, for large times and large deformations, there are modest qualitative differences for the two values of $\lambda$.

A second point that we consider, via the numerical results shown in Fig. 8, is the robustness of the behavior described above to variation in the details of the initial shape. In the results illustrated here, we show the time-dependent evolution of the drop shape for two quite dramatically different initial conditions, i.e.,

$$
r_{s}^{(1)}=1-0.285 P_{2}(\cos \theta)
$$

and case 1

$t=14.4$
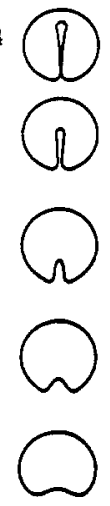

$t=0$

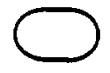

case 2
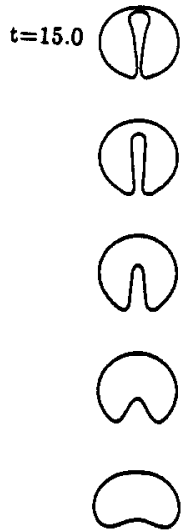

$\mathbf{t}=\mathbf{0}$

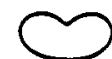

$\lambda=1.0$

FIG. 8. Comparison of two different initial conditions to show the robustness of the drop evolution to variation in the details of the initial shape. The time between intermediate shapes $=3.0$

$$
r_{s}^{(2)}=1-0.5 P_{2}(\cos \theta)-0.176 \mathrm{P}_{3}(\cos \theta),
$$

where the $P_{n}$ are the Legendre polynomials. The drop in the latter case has a very pronounced initial indentation on the front surface, though it is still globally oblate. The numerical solution shows that this indentation rapidly decays and again the upper surface of the drop reverts to a hemisphere. Beyond this, there is continued cavity growth at the rear of the drop, which is very similar to that obtained in the absence of the initial deformation.

One other interesting feature, which appears for at least one case when the drop develops a very elongated tail, is the appearance of a capillary wave instability that will presumably cause the tail to break into a large number of roughly equal-sized satellite drops. This phenomena is illustrated in Fig. 9, where we show the continuation of the case $\mathrm{Ca}=2.0$, $\Delta_{\text {initial }}=\frac{1}{3}$, and $\lambda=5.0$ (shown in Fig. 7) for larger times. It should be noted that this phenomenon was not observed for the deformation of prolate drops at lower viscosity ratios (i.e., $\lambda=0.1$ and $\lambda=0.5$ ). Evidently, the breakup of an initially unstable, prolate-shaped drop will occur by one of two possible mechanisms. When the drop is not very viscous, the tail thins dramatically at one point directly behind the parent drop, and we infer that pinch-off will occur in these cases in such a way that the drop breaks into two parts-the parent and a single satellite. On the other hand, as the tail elongates for a very viscous drop, pinch-off at a point is inhibited. As the drop continues to rise, it elongates and thins out more or less uniformly along the whole tail at the same time. Meanwhile, the amplitude of the capillary waves becomes finite, which eventually leads to breakup. This transition from pinch-off of the tail at a single point, to pinch-off at many points resulting from finite-amplitude capillary waves,

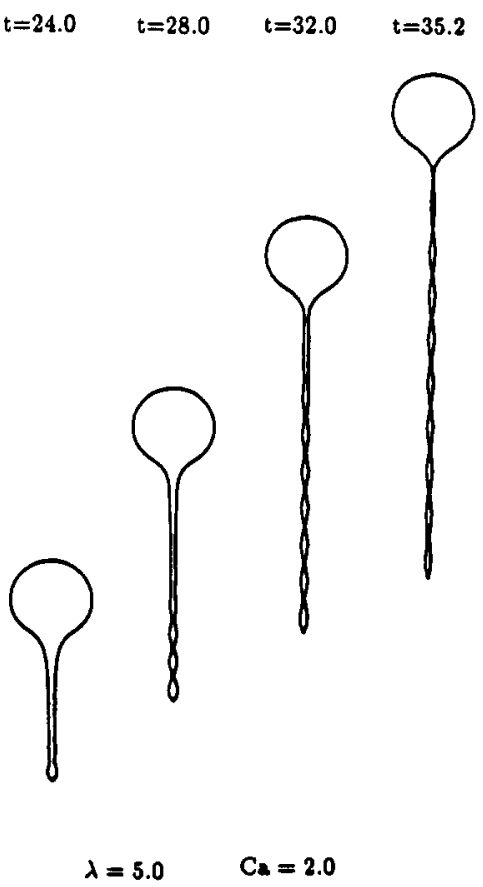

FIG. 9. Development of capillary waves on an elongated tail. 


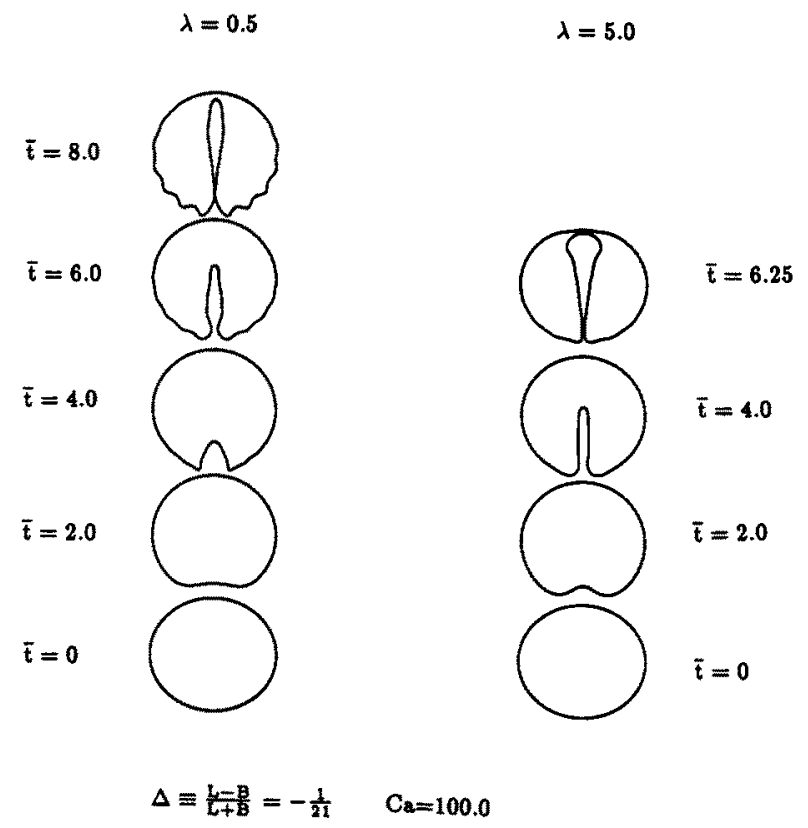

FIG. 10. The wavy structure is apparent on the less viscous drop $(\lambda=0.5)$, but the contrary on the more viscous drop $(\lambda=5.0)$. Note that the positions of the drops do not correspond to the exact numerically calculated displacement.

is highly analogous to the transition in breakup modes for an elongated drop in a quiescent fluid, from end pinching, in which a drop breaks by a sequence of single pinch-off extents at the ends of the drop, to a capillary wave breakup mechanism when the drop is more highly elongated (Stone and Leal ${ }^{3}$ ).

As it turns out, wavelike behavior is also present in the deformation of initially oblate drops. Although we have not made a comprehensive study, it is apparent, at least for the cases we studied, that viscous drops are less susceptible to the development of wavy shapes. Figure 10 compares the deformation of two drops with the same initial shape and capillary number, but different viscosity ratios. We can see that the wavy structure is quite pronounced for the less viscous drop but virtually nonexistent for the more viscous one. We should note that the development of these "waves" does not alter the overall qualitative behavior of the development of a cavity at the rear of the drops.

As stated initially, the primary objective of the study reported here is to establish the conditions for instability of an initially deformed drop at finite capillary number. The results reported above are summarized in Fig. 11. Here, we plot the lower and upper bounds of the critical value of $\mathrm{Ca}$ for several fixed values of $\Delta$. Results for three different $\lambda$

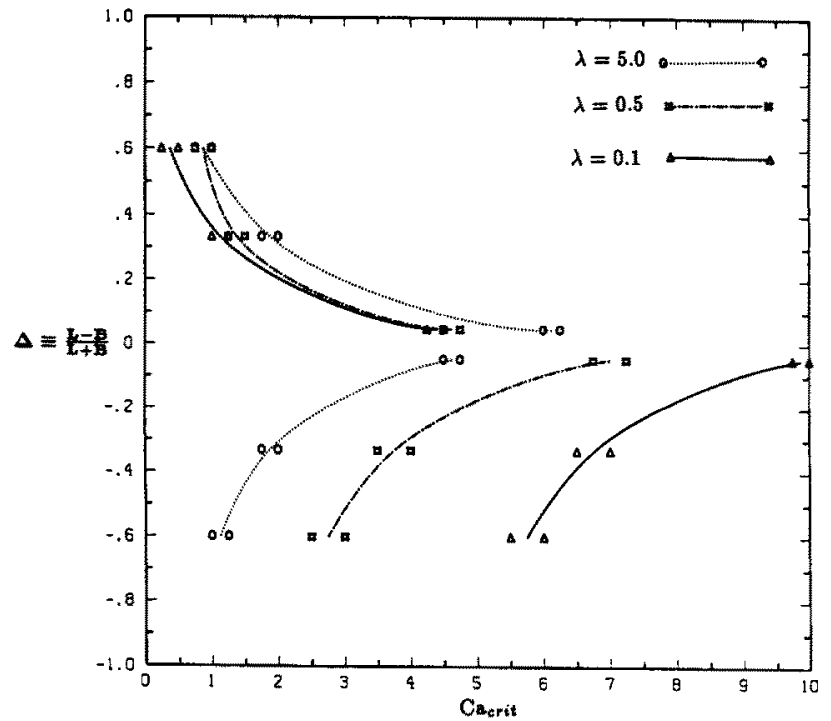

FIG. 11. Plot of $\mathrm{Ca}_{\text {crit }}$ vs $\Delta$ for both oblate and prolate drops. The symbols represent the lower and upper bounds of $\mathrm{Ca}_{\mathrm{crit}}$. The lines do not correspond to the exact value of $\mathrm{Ca}_{\text {crit }}$, but are drawn to show the trend.

values, namely, $0.1,0.5$, and 5.0 , are reported for both prolate and oblate initial shapes. There are several features that can be extracted from this figure. First, we can see that for he same initial shape, a more viscous prolate drop is more itable than a less viscous one (i.e., it has a higher $\mathrm{Ca}_{\text {crit }}$ ). However, the contrary is true for initially oblate drops. Second, it is apparent that the dependence of the $\mathrm{Ca}_{\text {crit }}$ on $\lambda$ is much smaller for prolate drops than for the oblate ones. Finally, this plot reemphasizes the point made earlier that drops at nonzero $\mathrm{Ca}$ are unstable to finite initial deformations, with the critical value of Ca increasing monotonically as $\Delta$ is decreased. This behavior seems qualitatively consistent with the predicted linear instability for the limit $\mathrm{Ca} \rightarrow \infty$.

\section{ACKNOWLEDGMENTS}

C. J. K. wishes to thank Dr. I. S. Kang for his helpful comments and discussion.

This work was supported by a grant from the Fluid Mechanics Program of the National Science Foundation.

'T. D. Taylor and A. Acrivos, J. Fluid Mech. 18, 466 (1964).

${ }^{2}$ M. Kojima, E. J. Hinch, and A. Acrivos, Phys. Fluids 27, 19 (1984).

${ }^{3}$ H. A. Stone and L. G. Leal, J. Fluid Mech. 198, 399 (1989).

${ }^{4}$ L. G. Leal, Computational Studies of Drop and Bubble Dynamics in a Viscous Fluid, Proceedings of the 3rd International Colloquium of Drops and Bubbles, Monterey, September 1988. 